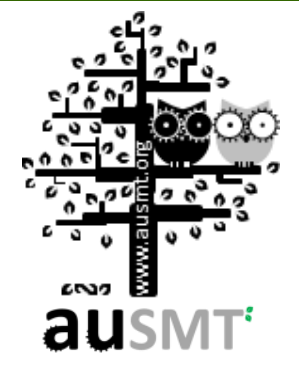

\title{
Fabrication of Sub-wavelength Antireflective Structure to Enhance the Efficiency of InGaAs Solar Cells
}

\author{
Yen-Yu Chou' ${ }^{1}$, Che-Chun Chang1, and Yeeu-Chang Lee ${ }^{2, *}$ \\ ${ }^{1}$ Department of Mechanical Engineering, Chung Yuan Christian University, Taiwan \\ ${ }^{2}$ Department of Mechanical Engineering, R\&D Center for Microsystem Reliability and Center of Biomedical Technology, Chung Yuan \\ Christian University, Taiwan \\ (Received 18 February 2014; Accepted 12 March 2014; Published on line 1 December 2014) \\ *Email: yclee@cycu.edu.tw \\ DOI: 10.5875 /ausmt.v4i3.623
}

\begin{abstract}
Large differences in the refractive index between semiconductors (Si, GaAs, etc.) and air produces considerable Fresnel loss, which can seriously hinder the absorption of sunlight by photovoltaic solar cells. This study presents a cost-effective roller nanoimprinting technique for the fabrication of sub-wavelength structures (SWSs) as an alternative to conventional anti-reflective coatings used to reduce reflectance in triple-junction InGaP/InGaAs/Ge solar cells. The proposed nanoimprinting technology uses a soft PDMS mold duplicated from a hard silicon template, which is fabricated using PS sphere lithography and dry etching processes. To evaluate the anti-reflective performance of SWSs, we employed rigorous coupled wave analysis (RCWA) to simulate the propagation of electromagnetic plane waves in a GaAs substrate. Simulation results demonstrated a considerable reduction in reflectance resulting from a gradual change in the refractive index provided by SWSs. Photoelectric conversion efficiency was also increased.
\end{abstract}

Keywords: Anti-reflective; sub-wavelength structure; RCWA; solar cell

\section{Introduction}

The poor photoelectric conversion efficiency (PCE) of Gallium arsenide (GaAs) based solar cells can be attributed to the large difference in the refractive indexes between air $(n=1)$ and GaAs $(n \approx 3.8)$, resulting in considerable Fresnel loss. Anti-reflective (AR) coatings can improve the PCE of solar cells by reducing surface reflection. Conventional single/multilayer AR thin films reduce surface reflection through destructive interference. Sub-wavelength structures (SWSs) have attracted considerable attention as replacements for conventional $A R$ thin films due to their broadband $A R$ performance. The refractive index of SWSs provides a graded alteration in the volume fraction of the structures, which in turn reduces Fresnel reflection. Various methods have been proposed to fabricate SWSs, including electron beam (EB) lithography, imprinting lithography, colloidal lithography, and laser interference lithography [1-4]. This study fabricated two dimensional hexagonal-close-packed SWSs on a triple-junction (InGaP/InGaAs/Ge) solar cell using a rapid, low cost process with excellent reusability $[5,6]$.

\section{Simulation and Experiments}

\section{Analysis of AR properties using the RCWA method}

To calculate anti-reflection performance, this study employed the rigorous coupled wave analysis (RCWA) method proposed by Lee et al. [7] to analyze the propagation of electromagnetic plane waves in a GaAs substrate patterned with SWSs [8]. Figure 1 presents a cross-sectional schematic view of a parabolic SWS array as well as the parameters used in the simulation. 


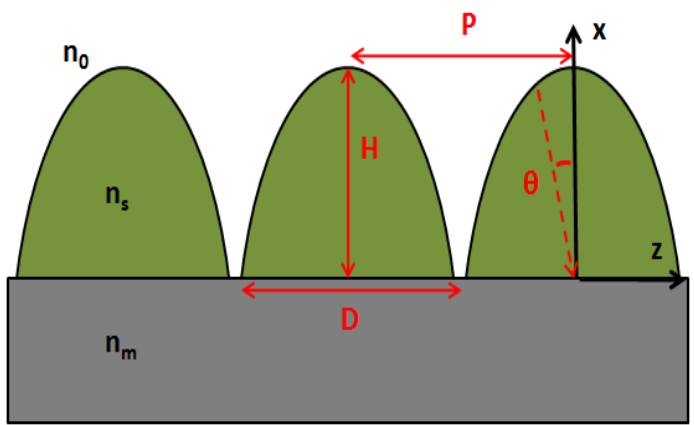

\begin{tabular}{cc}
\hline parameter & value \\
$\mathrm{s}$ & 1 (air) \\
\hline $\mathrm{n}_{0}$ & Varies with the wavelength \\
\hline $\mathrm{n}_{\mathrm{s}}$ & 1.9 (A54) \\
\hline $\mathrm{n}_{\mathrm{m}}$ & $550 \mathrm{~nm}$ \\
\hline$D$ & $390 \mathrm{~nm}$ \\
\hline $\mathrm{H}$ & $550 \mathrm{~nm}$ \\
\hline $\mathrm{P}$ & $300^{\sim} 1200 \mathrm{~nm}$ \\
\hline$\lambda$ & $5^{\circ}$ \\
\hline$\theta$ &
\end{tabular}

Figure 1. Cross-sectional schematic of SWS array and the parameters used in simulation.
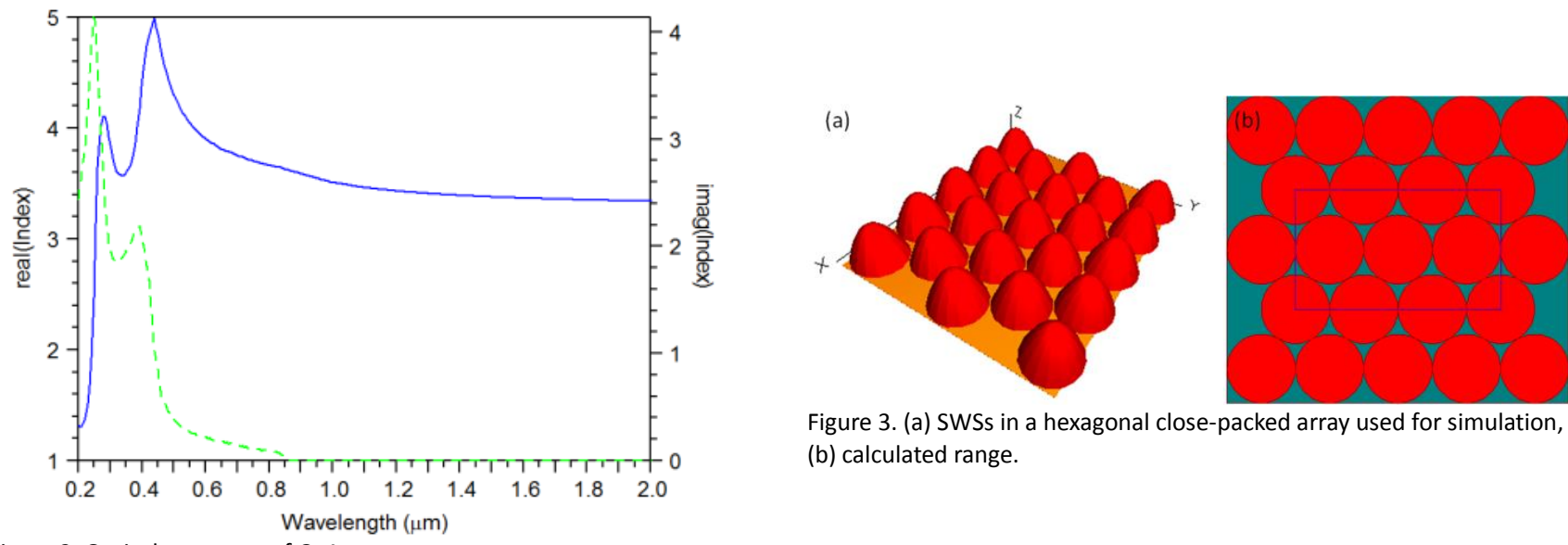

Figure 3. (a) SWSs in a hexagonal close-packed array used for simulation, (b) calculated range.

Figure 2. Optical constants of GaAs.
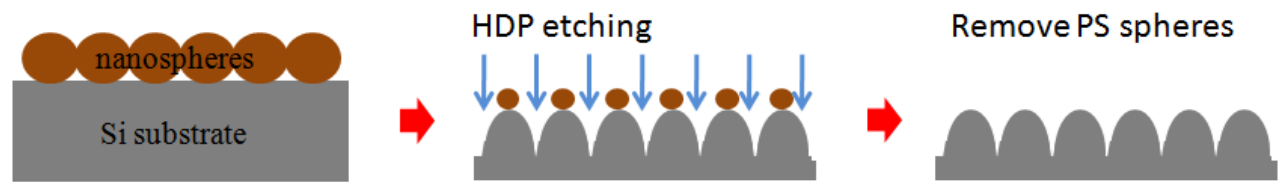

Figure 4. Schematic illustration of fabrication process of the silicon template.

Parameters $\mathrm{P}, \mathrm{H}, \mathrm{D}$, and $\mathrm{d}$ respectively represent the period, height, bottom diameter, and top diameter of the structures. $\theta$ is the incident angle of light, and $n_{s}$ and $n_{m}$ are respectively the optical constants (refractive index and extinction coefficient of the material) of the structure and substrate. In this study, $\mathrm{n}_{\mathrm{s}}$ is dependent on the wavelength of incident light, as shown in Figure 2 and $\mathrm{n}_{\mathrm{m}}$ is a constant with a value of 1.9. The geometrical

Yen-Yu Chou is a Ph.D. candidate at Chung Yuan Christian University. His research interests include ray tracing and rigorous coupled wave analysis (RCWA) simulation methods.

Che-Chun Chang received his M.S. from the Department of Mechanical Engineering, Chung Yuan Christian University, Chung Li, Taiwan, in 2012.

Yeeu-Chang Lee received his Ph.D. in mechanical engineering from National Central University, Chung Li, Taiwan, in 1998, respectively. In 2000, he was a Project Manager at HC Photonics, Inc., where he was engaged in the development of nonlinear optic chips. In 2004, he was a Senior System Engineer at Unaxis Taiwan Ltd., Hsinchu, Taiwan. He is currently an Assistant Professor in the Department of Mechanical Engineering, Chung Yuan Christian University. His research interests include nano/micron fabrication, optomechatronics system, and optoelectronic devices. structure of the modeled SWSs and the calculated range (unit cell) are respectively shown in Figures 3(a) and 3(b). To facilitate comparison with measured results, all experimental parameters were duplicated in the simulation.

\section{Fabrication of SWSS}

\section{Fabrication of PDMS mold}

Figure 4 outlines the Si template fabrication process. Polystyrene (PS) spheres with a diameter of 600 $\mathrm{nm}$ were uniformly sprayed onto a Si substrate to serve as a mask during the high-density plasma (HDP, Unaxis $\mathrm{GmbH}$, Nextral 860L) etching. During the HDP process, a mixture of $\mathrm{Ar}, \mathrm{SF}_{6}$, and $\mathrm{O}_{2}$ gasses were respectively introduced at flow rates of $25 \mathrm{sccm}, 50 \mathrm{sccm}$, and 40 $\mathrm{sccm}$. The pressure was held at $24.8 \mathrm{mTorr}$ for a period of $5 \mathrm{~min}$, whereupon the remaining PS spheres were removed using $\mathrm{O}_{2}$ plasma etching. 


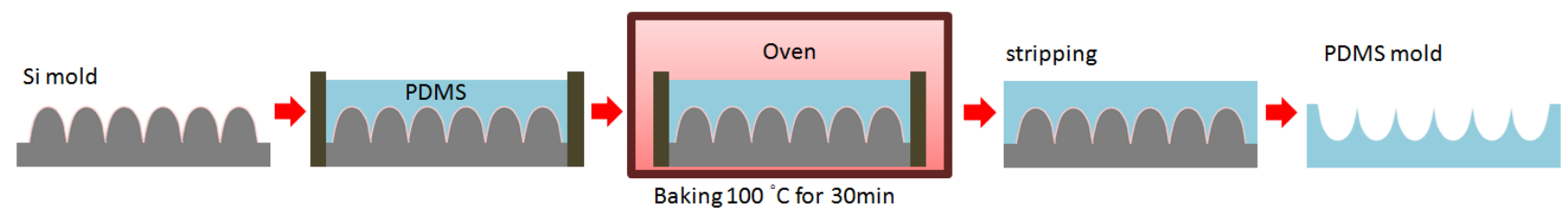

Figure 5. Schematic illustration of fabrication process of the PDMS mold.

This study employed a soft polymer PDMS (Polydimethylsiloxane) mold to duplicate the patterns from the Si template. This fabrication process has the advantages of rapid production, simplicity, and low fabrication costs. Moreover, for non-planar patterns, a flexible mold produces a higher quality imprint. Figure 5 presents the fabrication procedures for the PDMS mold. PDMS solvent, curing agent, and silicone oil were mixed (10:1:1 weight ratio) and degassed in a vacuum chamber to remove air bubbles. After curing at $100{ }^{\circ} \mathrm{C}$ for $30 \mathrm{~min}$, the solidified PDMS mold was peeled away from the $\mathrm{Si}$ template.

\section{Roller imprinting}

Roller imprinting was used to produce SWSs on the surface of InGaP/InGaAs/Ge triple junction solar cells, as shown in Figure 6.

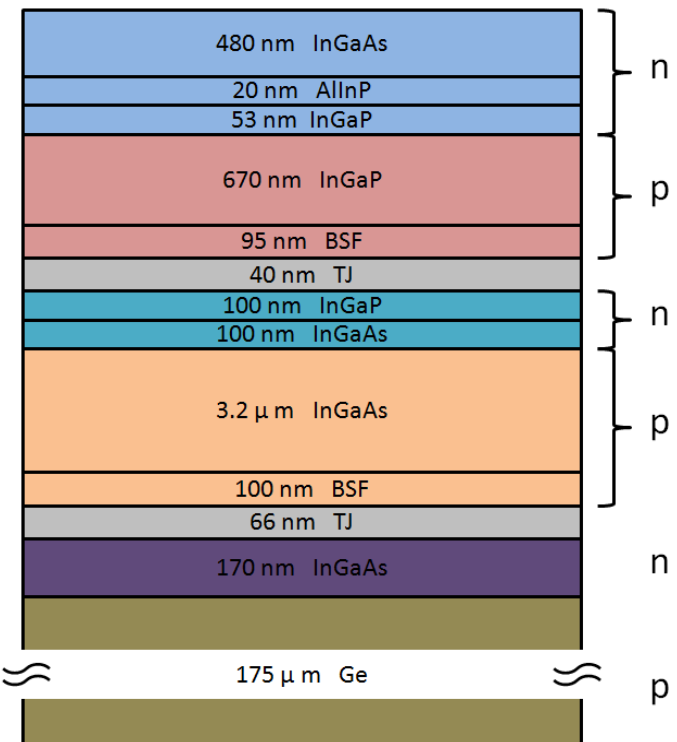

Figure 6. Cross-sectional view of the $\operatorname{lnGaP} / \mathrm{InGaAs} / \mathrm{Ge}$ triple junction solar cell.

The PDMS soft mold was first sprayed with a release agent (SR-5764) at $2000 \mathrm{rpm}$ for $30 \mathrm{~s}$ before being cured at $110^{\circ} \mathrm{C}$ for $2 \mathrm{~min}$. This helped to reduce the surface energy and make it easier to remove the imprinted material from the Si mold. The refractive index of the imprinted materials was 1.9 (OptiNDEXTMA54, Brewer Science Inc.). The imprinting material was spun onto the PDMS mold at $3000 \mathrm{rpm}$ for $40 \mathrm{sec}$ at room-temperature. The solar cell was fixed to the translation stage using a vacuum holder, and the imprinted material was transferred from the PDMS mold onto the solar cell surface using roller imprinting equipment, as illustrated in Figure. 7.

Following the imprinting process, the electrical properties of the solar cell were characterized under simulated AM1.5G illumination at room temperature and at a power of $100 \mathrm{~W} / \mathrm{m}^{2}$.

\section{Results and Discussions}

\section{Reflectance simulation of SWSs}

Figure 8 presents the reflectance spectra of a bare GaAs wafer and a GaAs wafer with A54 SWSs in the range of $300 \mathrm{~nm}-1200 \mathrm{~nm}$. The simulated spectrum of the bare GaAs wafer was highly similar to that observed in the experiment. Therefore, simulations are reliable as analytical instruments prior to experimentation. Although the refractive index of A54 (1.9) is far below that of GaAs, the influence of SWSs on reflectance is still obvious. The average reflectance of bare GaAs (35.0\%) was reduced to $13.9 \%$ by the application of SWSs.

SWS arrays perform like a stack of multi-layer thin films, in which the refractive index of each layer gradually changes due to changes in the volume fraction provided by the shape of the structures. The gradient in the refractive index was deduced using effective medium theory [9]. By dividing the height of the SWSs into several thin layers, an optical multilayer model was used to calculate reflectance as a function of height. Figure 9 illustrates how the effective refractive index varied according to the height of the A54 structures.

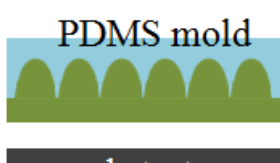

substrate

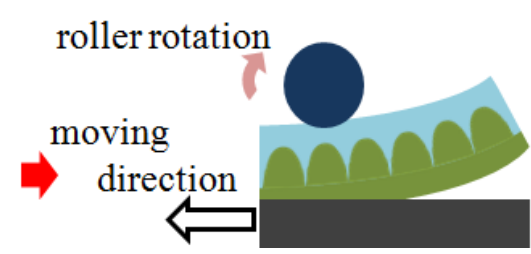

Figure 7. Schematic illustration of the roller imprinting process.

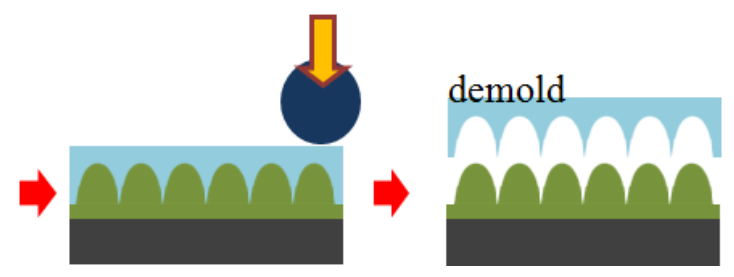

ausMT Vol. 4 No. 3 (2014) 


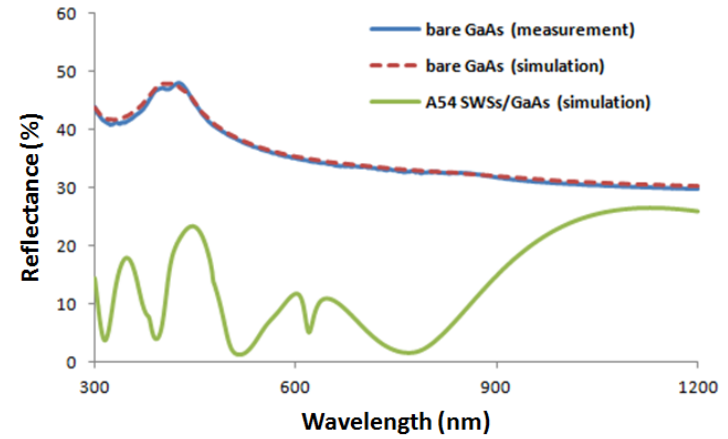

Figure 8. Reflectance spectra of bare GaAs and A54 SWSs/GaAs.

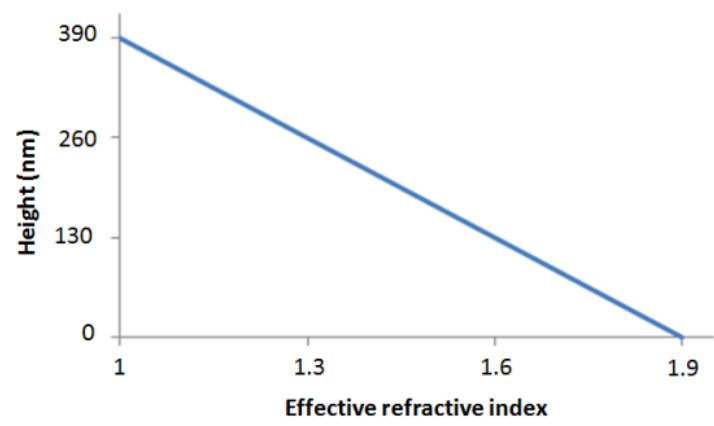

Figure 9. Variations in effective refractive index according to the height of paraboloid structures.

\section{Imprinting SWSs on triple-junction (InGaP/InGaAs/Ge) solar cell}

Figure 10 illustrates the HDP etching of Si wafers using PS spheres as a mask to fabricate SWSs. The paraboloidal structures were assembled in a close-packed arrangement at a height of approximately $600 \mathrm{~nm}$.
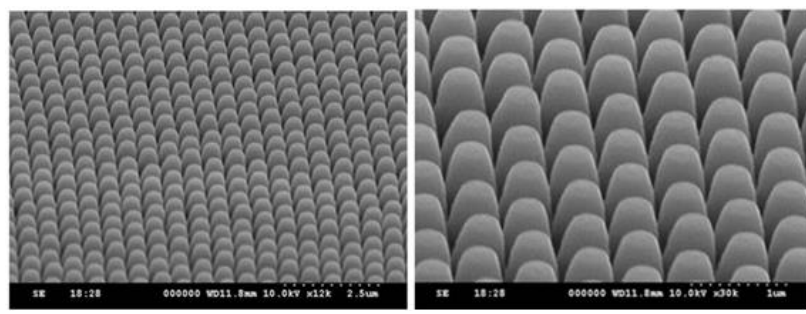

Figure 10. SEM images of Si template.

Figure 11 shows the PDMS mold made from the $\mathrm{Si}$ template. The surface structure of the PDMS lacks uniformity because the viscosity of the PDMS prevented the solution from flowing completely into the $\mathrm{Si}$ structures. This also reduced the depth of the holes to approximately $400 \mathrm{~nm}$.

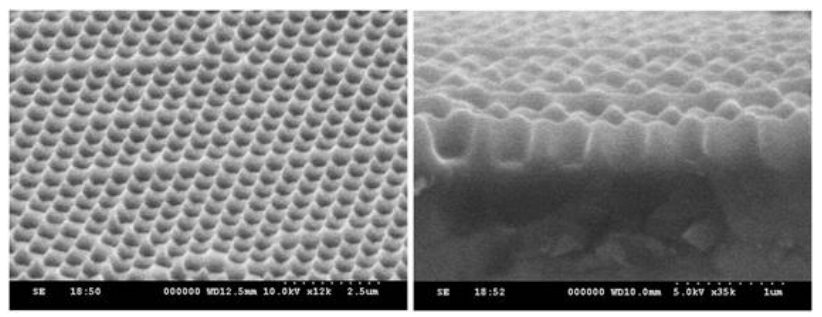

Figure 11. SEM images of PDMS mold.

www.ausmt.org

Copyright (C) 2014 International Journal of Automation and Smart Technology
Figure 12 shows the imprinted paraboloidal structures on the triple-junction (InGaP/InGaAs/Ge) solar cell. The refractive index of the imprinted material is 1.9 and the height of the imprinted structure is approximately $390 \mathrm{~nm}$. The imprinting pressure and imprinting speed were $300 \mathrm{kgf} / \mathrm{cm}^{2}$ and $2 \mathrm{~mm} / \mathrm{s}$, respectively. Although the imprinted structures are not as closely packed and uniform as the Si structures, a reduction in reflectance is still expected due to the gradual change in the refractive index produced by the SWSs.

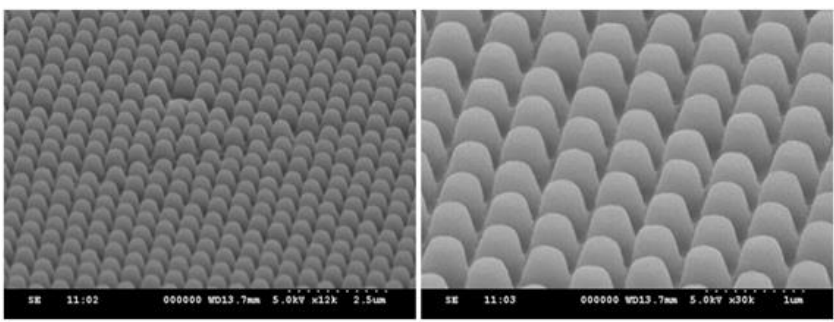

Figure 12. SEM images of imprinting structures.

The electrical properties of the solar cell were characterized using simulated AM1.5G illumination at room temperature at a power of $100 \mathrm{~W} / \mathrm{m}^{2}$, as shown in Table 1. The power conversion efficiency of the SWSs cell is $24.4 \%$, indicating a $5.2 \%$ improvement over the cell without SWSs.

Table 1. Current-voltage characteristics of planar and SWSs solar cell.

\begin{tabular}{ccccc}
\hline & $\mathrm{V}_{\text {oc }}(\mathrm{V})$ & $\mathrm{I}_{\mathrm{sc}}(\mathrm{mA})$ & $\begin{array}{c}\text { Filling } \\
\text { factor }(\%)\end{array}$ & $\begin{array}{c}\text { Efficiency } \\
(\%)\end{array}$ \\
\hline $\begin{array}{c}\text { Planar } \\
\text { cell }\end{array}$ & 2.4 & 3.4 & 84.5 & 23.2 \\
$\begin{array}{c}\text { SWSs } \\
\text { cell }\end{array}$ & 2.4 & 3.9 & 79.6 & 24.4 \\
\hline
\end{tabular}

\section{Conclusion}

Nanoimprinting was successfully applied to the fabrication of sub-wavelength structures on a triple-junction InGaP/InGaAs/Ge solar cell. Rigorous coupled wave analysis (RCWA) was used to simulate the propagation of electromagnetic plane waves in a GaAs substrate with SWSs. According to simulation results, the average reflectance of bare GaAs (35.0\%) (refractive index $=1.9$ ) was reduced to $13.9 \%$ by the application of SWSs. Using a solar simulator to produce the standard solar spectrum (AM 1.5G) with a power of $100 \mathrm{~W} / \mathrm{m}^{2}$, the power conversion efficiency of the SWS cell was $5.2 \%$ higher than that of the cell without SWSs. 


\section{References}

[1] Q. Xie, M. H. Hong, H. L. Tan, G. X. Chen, L. P. Shi, and T. C. Chong, "Fabrication of nanostructures with laser interference lithography," Journal of Alloys and Compounds, vol. 449, no. 1-2, pp. 261-264, 2008.

doi: 10.1016/j.jallcom.2006.02.115

[2] K. S. Cho, P. Mandal, K. Kim, I. H. Baek, S. Lee, H. Lim, D. J. Cho, S. Kim, J. Lee, and F. Rotermund, "Improved efficiency in gaas solar cells by $1 \mathrm{~d}$ and 2d nanopatterns fabricated by laser interference lithography," Optics Communications, vol. 284, no. 10-11, pp. 2608-2612, 2011. doi: 10.1016/i.optcom.2011.01.042

[3] S.-H. Hong, B.-J. Bae, J.-Y. Hwang, S.-Y. Hwang, and $\mathrm{H}$. Lee, "Replication of high ordered nano-sphere array by nanoimprint lithography," Microelectronic Engineering, vol. 86, no. 12, pp. 2423-2426, 2009. doi: $\underline{10.1016 / \text { i.mee.2009.05.005 }}$

[4] H. L. Chen, S. Y. Chuang, C. H. Lin, and Y. H. Lin, "Using colloidal lithography to fabricate and optimize sub-wavelength pyramidal and honeycomb structures in solar cells," Optics Express, vol. 15, no. 22, pp. 14793-14803, 2007. doi:10.1364/OE.15.014793

[5] J. Lee, S. Park, K. Choi, and G. Kim, "Nano-scale patterning using the roll typed uv-nanoimprint lithography tool," Microelectronic Engineering, vol. 85, no. 5-6, pp. 861-865, 2008. doi: $10.1016 /$ j.mee.2007.12.059
[6] C. J. Ting, C. F. Chen, and C. P. Chou, "Subwavelength structures for broadband antireflection application," Optics Communications, vol. 282, no. 3, pp. 434-438, 2009.

doi: $10.1016 /$ i.optcom.2008.10.026

[7] Y. C. Lee, C. C. Chang, and Y. Y. Chou, "Experimental and simulation studies of anti-reflection sub-micron conical structures on a gaas substrate," Optics Express, vol. 21 Suppl 1, pp. A36-41, 2013. doi: $10.1364 /$ oe.21.000a36

[8] N. Yamada, O. N. Kim, T. Tokimitsu, Y. Nakai, and H. Masuda, "Optimization of anti-reflection moth-eye structures for use in crystalline silicon solar cells," Progress in Photovoltaics: Research and Applications, vol. 19, no. 2, pp. 134-140, 2011. doi: $10.1002 /$ pip.994

[9] D. G. Stavenga, S. Foletti, G. Palasantzas, and K. Arikawa, "Light on the moth-eye corneal nipple array of butterflies," Proceedings of The Royal Society B: Biological Sciences vol. 273, no. 1587, pp. 661-667, 2006.

doi: $10.1098 \% 2 \mathrm{frspb} .2005 .3369$ 